\title{
Influence of crystalline structure on rf dissipation in superconducting niobium
}

\author{
C. Z. Antoine \\ CEA, Irfu, SACM, Centre d'Etudes de Saclay, 91191 Gif-sur-Yvette Cedex, France
}

(Received 15 June 2018; published 7 March 2019)

\begin{abstract}
Bulk niobium is the most common material used in the fabrication of rf superconducting cavities for accelerators. Predicting and reducing the rf surface dissipation in these cavity structures is mandatory, since it has a tremendous cost impact on the large accelerator projects. In this paper the author hopes to demonstrate that sources of dissipation usually attributed to external causes (mainly flux trapping during cooldown and hydrides precipitates) are related to the same type of crystalline defects that affect the local superconducting properties and can be at the source of early vortex penetration at the surface. We also want to show how these types of defects can explain some of the discrepancies observed from other laboratories in niobium cavity doping experiments. Understanding the origin and the role of these defects could provide direction for improving material specifications as well as improving fabrication control from sheet material to completed cavity. In particular, we will demonstrate that dislocation entanglements, due to the fabrication damage layer, have the strongest impact for the pinning behavior of trapped flux, as well as hydrogen segregation in cavity niobium. The author wishes to present to the superconducting radio frequency (SRF) accelerator community the synthesis of experimental results scattered in the literature, completed with some personal results. The results of this effort provide a new perspective on recently published work in the domain of SRF cavity doping and sensitivity to trapped flux during cooldown. I will also try to draw whenever possible, some conclusions about other types of superconductors used for SRF applications including $\mathrm{Nb} / \mathrm{Cu}$ thin films and to discuss their possible change of behavior with field or frequency. I will concentrate on surface and material science aspects since the experimental results on rf cavities have already been treated elsewhere.
\end{abstract}

DOI: 10.1103/PhysRevAccelBeams.22.034801

\section{INTRODUCTION}

Most of the present models for determining the surface resistance of superconductors in the rf regime (SRF) do not take into account the most common crystalline defects found in bulk niobium. Indeed, models that allow the user to evaluate rf surface resistance (Mattis and Bardeen integrals) are models based on the assumption that the density of superconducting electron, $n_{s}$, is uniform [1]. During the development of superconducting (SC) material for magnets, it has been amply demonstrated that many phenomena are related to the fact that ns is not uniform [2]. Ginsburg-Landau (GL) developments that account for some nonuniformity and nonlinear behavior are abundant in the literature, but they are valid only close to the critical temperature $\mathrm{T}_{C}$ or the second critical field $\mathrm{H}_{C 2}$, unless one

*claire.antoine@cea.fr

Published by the American Physical Society under the terms of the Creative Commons Attribution 4.0 International license. Further distribution of this work must maintain attribution to the author(s) and the published article's title, journal citation, and DOI. deals with linearized GL model that applies at any T, but only for very small coherence length $\xi$. The majority of the numerical developments found in the literature have been conducted for high Ginzburg-Landau parameter $\kappa$ (small $\xi$ ) superconductors [3-5]. Their numerical predictions are generally poor for niobium with its large $\xi(\sim 40 \mathrm{~nm})$ and $\kappa$ close to 1 , especially in the SRF operating condition (Meissner state, 2-4 K). Recent models proposed by the SRF community start to introduce some defects and nonuniformity, but in most of the models, defects are considered to be uniformly distributed, which is not a fully realistic description [6-8].

In this paper, the author hopes to demonstrate that the same type of defects are at the origin of pinning behavior as well as hydrogen segregation, and that it influences the atomic diffusion related phenomena.

\section{A. Crystalline defects}

From the metallurgical point of view, defects like dislocation or interstitial atoms do not distribute evenly inside the lattice, but tend to appear and/or diffuse along specific crystalline orientations $[9,10]$. Moreover, since some defects interact together, they tend to gather in 


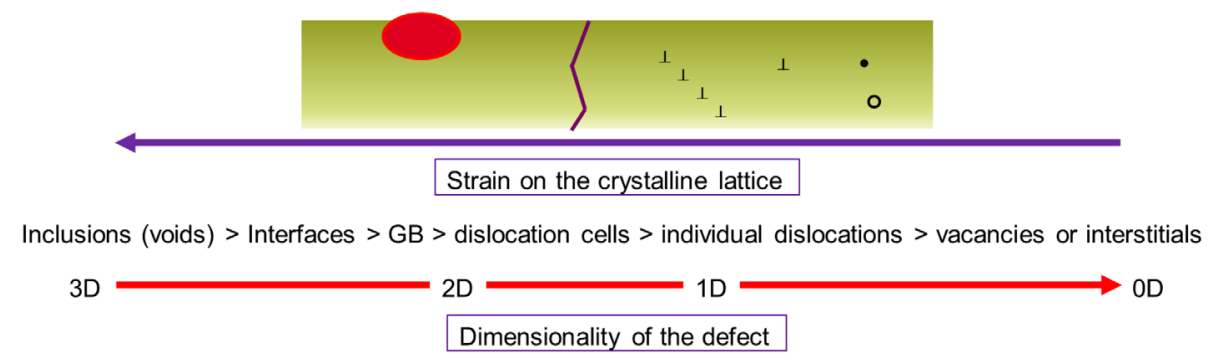

FIG. 1. Typical defects found in crystalline structures. The strain increases with dimensionality of defects; it affects atomic diffusion behavior and local SC properties like $\xi$ or mean-free path as well (see text below for more details).

specific crystalline regions, e.g., at grain boundaries, or at subgrain levels. Most of these defects induce an elastic deformation of the lattice (Fig. 1), which can be quantified in some cases, and that has a large impact on the atomic diffusion behavior such as interstitial atoms diffusion implied in doping, or dislocation movement implied in deformation and/or annealing effects.

Those defects are as well at the source of the local variations of the SC parameters, including the local density of Cooper pairs $n_{s}$ and thus on their pinning behavior (see below for more details). The relation between pinning and crystalline defects has been thoroughly studied and as it affects strongly the trapping of magnetic flux upon cooldown, we will detail it in the following section.

\section{B. Generalities on pinning}

Pinning behavior of crystalline defects has been thoroughly studied from the theoretical point of view during the development of SC cable (see textbooks, e.g., [3,4,11]); a lot of data can be found on $\mathrm{Nb}$, bulk as well as thin films in the literature (e.g., [12-16]). The detail of the physical phenomena implied in the pinning behavior can also be used to explain the very type of behavior observed on cavities, such as hydride precipitates or lab to lab performance variability.

Matsushita [4] describes four main mechanisms involved in pinning behavior. The relative weight of each mechanism depends on the defect types as well on the SC properties of the considered material.

Condensation energy gain.-Making vortices go through a normal conducting area (or at least an area with depressed superconductivity) results in a gain in Gibbs free energy. At equilibrium, it is counterbalanced by the energy necessary to elastically deform the vortices lattice. This mechanism is dominant in the case of normal conducting precipitates.

Elastic interactions.- The elastic modulus in the superconducting state is always less than in the normal state [17]. Moreover, the relative elastic modulus difference between normal and SC state, $\Delta \mathrm{Y} / \mathrm{Y}$, is known to decrease with decreasing mean-free path [13]. Since crystalline defects also modify the local elastic properties of the lattice, the elastic interaction might become the dominant aspect in pinning (see Sec. III).

Magnetic interaction.-If the defect's size is larger than $\lambda$, one can treat it like an interface energy barrier analogous to the surface barrier between the vacuum-superconductor interface with image vortex formalism [18].

Kinetic interaction.-Areas with different $\xi$ exhibit different vortex velocities, resulting in local nonuniformity that is not taken into account in the simple London model (two fluid approach).

In a general way, pinning results from local lattice defects and local variation in the SC parameters, and there are several ways to achieve strong pinning: (i) "surface magnetic pinning" where the main mechanism is magnetic pinning resulting from large defects: twinning, voids, non-SC aggregates, irradiation defects (columnar), nanoindentations. In this case the dominant mechanism is condensation energy gain and magnetic interaction. The maximum pinning force occurs when the larger dimension of the defect is parallel to the flux line and its diameter $\mathrm{d}$ is close to $2 \xi$. Overall, if $\mathrm{d}$ is $\ll$ to $2 \xi$, then the gain in condensation energy is negligible, whereas when $\mathrm{d}>2 \xi$, no further gain can be expected for one single vortex (but then several vortices can pin on a large defect). An example of such a strong pinning center would be normal conducting $\mathrm{Ti}$ precipitates in NbTi wires [19]. (ii) "Volume core pinning" with individual weak pinning centers, many of them (e.g., interstitials or small precipitates evenly distributed in the material; individual dislocations). The main mechanism would be elastic interaction, which is much less efficient than the magnetic interaction, but if there are many pinning centers it results into very strong pinning nonetheless. An example of many weak pinning centers resulting in efficient collective pinning would be oxygen vacancies in $\mathrm{Bi}-2212$ [20].

\section{PINNING IN NIOBIUM}

\section{A. General observations}

Pinning is at the origin of irreversibility in dc magnetization curves, as the surface inside the hysteresis loop of a magnetization curve is directly proportional to the pinning force [21]. As it can be seen in Fig. 2 (after [22]), irreversibility is also related to the crystalline state of the 

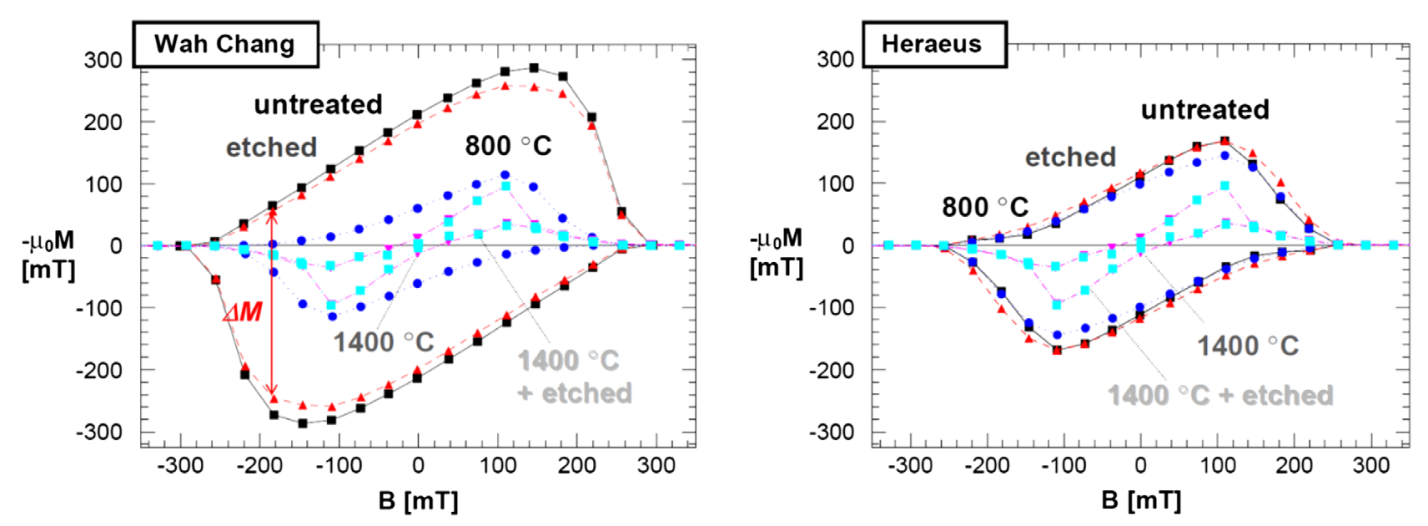

FIG. 2. dc magnetization curves from two different $\mathrm{Nb}$ batches (modified from [22]). At a given field, within the bean model, $\Delta \mathrm{M}$ is proportional to the critical current $\mathrm{J}_{C}$, as well to the pinning force $\mathrm{F}_{P}$ [26]. One can observe that recrystallization at $800{ }^{\circ} \mathrm{C}$ and even better at $1400{ }^{\circ} \mathrm{C}$ decreases the hysteresis in the curve, indicating a decrease in pinning efficiency. Unless the sample is a perfect ellipsoid, it is not possible to make the hysteresis totally disappear, because of surface pinning and edge pinning.

material, and recrystallization can improve greatly the reversibility of the magnetization curve. Effects of damage layer and/or alloying on ac losses of $\mathrm{Nb}$ have been explored in [23] where it is shown that ac losses can be directly inferred from the dc magnetization curve. In some cases, pristine surfaces exhibit a lot of flux jumps characteristic of surface pinning due to the damage layer [24]. Note that in dc magnetometry it is difficult to get uniform field at the surface of the sample. Demagnetization effect and surface pinning need to be considered, which explains why even after $1400{ }^{\circ} \mathrm{C}$ treatment the samples seem still to exhibit some hysteresis. An experiment conducted on perfect ellipsoids shows that $1400^{\circ} \mathrm{C}$ baking virtually eliminates all pinning in niobium [25].

Since high temperature treatments have a detrimental effect on the mechanical integrity of bulk niobium, one has to consider that some flux trapping will always occur. Therefore, it is also important to determine which kind of crystalline defects is more detrimental to SRF applications. Indeed, many classical publications on pinning deal with high $\kappa$ material (large $\lambda$, small $\xi$ ), since it is the case for all the superconductors used for magnet developments, where pinning plays a large role. In the case of niobium, with $\kappa \sim 1$ some of the numerical approximations do not hold anymore and relative pinning forces need to be reconsidered [27].

\section{B. Surface pinning}

Surface pinning was not considered until recently in SRF, but there are several experimental indications that it might play an important role. For instance, Pautrat et al. studied the surface pinning on corrugated $\mathrm{Nb}$ samples in relation to roughness [28]. They showed that increasing roughness increases the critical current (which is proportional to flux pinning), but they also showed that since the distance between vortices changes with field, the range of roughness to be considered changes too. Indeed, at low field one is mainly sensitive to defects around $0.1-1 \mu \mathrm{m}$ while at higher field one is sensitive to smaller scale roughness. It is believed that roughness far below the $\xi$ range does not play a role. The explanation for such a high effect of surfaces/interfaces results from the limit condition: $\vec{J}_{S} \times \vec{n}=\overrightarrow{0}$, which obliges the vortices to exit the superconductor always perpendicular to the surface. If that surface has a certain roughness, it results in bending of the vortex, which increases its length, which in turn increases the elastic return force. As pinning and elastic forces are in equilibrium, hence the pinning force also automatically increases. This effect is mainly observed on thin films where the surface/volume ratio is high [28], and it could play a paramount role for alternative superconductors $\left(\mathrm{NbN}, \mathrm{Nb}_{3} \mathrm{Sn}, \mathrm{MgB}_{2}, \ldots\right)$ in SRF applications: most of these materials are fabricated into relatively thin films with relatively small grain sizes at the origin of some roughness at the $\mathrm{nm}$ level. One has still to verify that the impact of surface nanoroughness is negligible in SRF applications.

A similar rationale (flux line should exit perpendicular to the surface) applies at higher scale to edge effects in magnetometry [28-30]. It might also play a role for a nearly perfect material where little or no volume pinning occurs and probably can explain some of the $\mu \mathrm{SR}$ results observed recently on nonmasked samples (e.g., in [31]) and that will be discussed below.

\section{Effect of frequency: Bulk vs thin films}

A very simple technique is widely used to determine the volume pinning force $[32,33]$; it consists in measuring the complex impedance of samples in order to determine the complex penetration depth $\lambda_{\mathrm{AC}}=\lambda^{\prime}+\mathrm{i} \lambda^{\prime \prime}$ in an ac setup as shown in Fig. 3. The measured flux is proportional to $\lambda_{\mathrm{ac}}: \phi_{a c}=\int b_{a c} d S \sim 2 \lambda_{a c} \ell_{b} b_{0}$.

At low frequency, $\lambda^{\prime}$ is proportional to $\delta_{\mathrm{ac}}$ (penetration depth in normal state) since the flux fully enters the superconductor and $\lambda^{\prime \prime} \sim 0$ (magnetic screening is inefficient). Since the vortices are efficiently pinned at low 


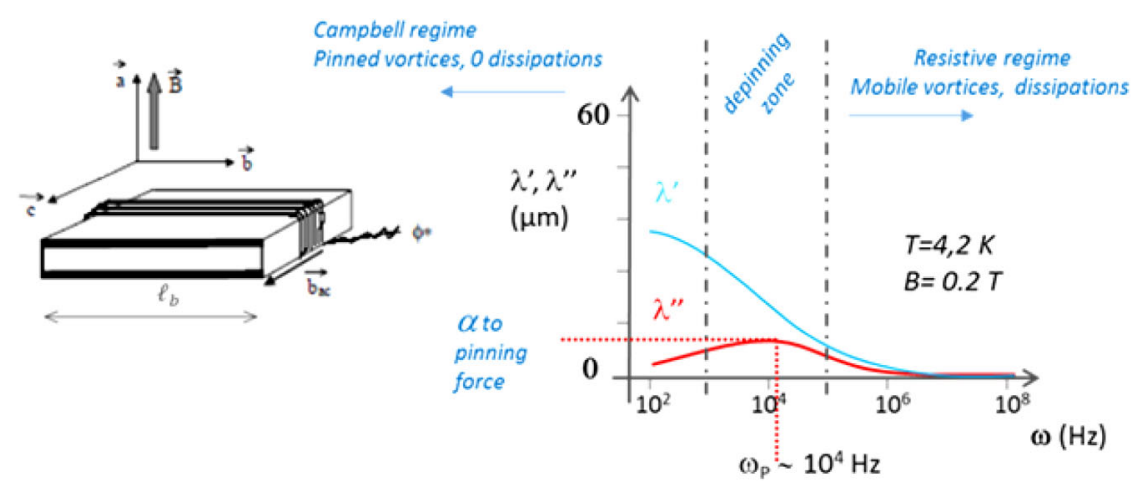

FIG. 3. Left: Principle of measuring ac complex penetration depth. The sample is immersed in a dc field B perpendicular to the sample surface $(/ / \vec{a})$. An additional ac field is applied to the sample via the coil around the sample. In this configuration currents are mainly confined in the $(\vec{b}, \vec{c})$ direction. The measured flux $\phi_{a c}=\int b_{a c} d S \sim 2 \lambda_{a c} \ell_{b} b_{0}$. At low frequency $\lambda^{\prime} \sim \delta_{R F}$ (penetration depth in normal state). At high frequency $\lambda^{\prime} \sim \lambda^{\prime \prime} \sim \lambda_{L} \ll \delta_{\text {rf }}$. Right: Results obtained for high quality Nb monocrystal (modified from [32]).

frequency, the resistance Rs is strictly 0 (so-called Campbell regime).

At high frequency, pinning is inefficient and $\lambda^{\prime} \sim \lambda^{\prime \prime} \sim$ $\lambda_{L} \ll \delta_{\text {rf }}$. The surface resistance follows the usual $\mathrm{R}_{\mathrm{BCS}}+$ $\mathrm{R}_{\mathrm{Res}}$ behavior, where $R_{\mathrm{BCS}}$ refers to the the temperature dependant surface resistance as predicted by the Bardeen Cooper Schrieffer (BCS) theory and $R_{\text {Res }}$ gathers other phenomena independent of $\mathrm{T}$.

The maximum of $\lambda^{\prime \prime}$ is proportional to the pinning force and defines the so-called depinning frequency. Note that critical currents are also a good way to evaluate the effective pinning force.

Figure 3 shows the results for bulk monocrystalline high quality $\mathrm{Nb}\left(\mathrm{RRR}^{1} \sim 5000\right)$ after Ref. [32]. The depinning frequency lies around $10^{4} \mathrm{~Hz}$, which shows that it is very unlikely that pinning would still persist at SRF frequency in the absence of crystalline defects. The fact that higher depinning frequency $(10 \mathrm{MHz}-1 \mathrm{GHz})$ has been observed in SRF $\mathrm{Nb}$ [34] is clear evidence that SRF niobium retains crystalline defects that act as pinning centers.

The same type of experiment applied to thin film $\mathrm{Nb}$ deposited with various techniques shows that depinning frequency varies a lot with the quality of the films, but is typically ranging in the $10-30 \mathrm{GHz}$ interval [35-38]. This explains why thin films show low sensitivity to trapped magnetic flux: nearly all the flux is efficiently trapped, and those superconductors are kept in the Campbell regime. In this configuration, it is possible that some vortex starts to detrap as the field increases, explaining the slope $\mathrm{dRs} / \mathrm{dB}$ observed on thin films. See also [39,40] for theoretical support and [41] for recent SRF results.

In the next paragraph, we will describe generalities about flux trapping and in the following paragraphs we will describe crystallographic defects commonly found inside $\mathrm{Nb}$ and try to determine the main relevant pinning mechanism for SRF grade niobium.

\footnotetext{
${ }^{1}$ RRR: Residual Resistivity Ratio.
}

\section{Flux trapping during cooldown}

Flux trapping originates mainly from the trapping of residual (earth or thermocurrent induced) magnetic field when cooling down of the cavities. Early work from Turneaure and Weisman [42] showed that it was possible to get very high Q0 for an electron beam melted $\mathrm{Nb}$ TE011 cavity at $11 \mathrm{GHz}$ in a magnetically shielded cryostat. More systematic studies started in the 1990s, when it was shown that thin film cavities and bulk behaved differently, thin film being apparently insensitive to external magnetic flux [43]. During his Ph.D. (1994), Valet showed for the first time that cooling down in field ranging from a few $\mathrm{mG}$ to some hundreds $\mathrm{mG}, 100 \%$ of the field was trapped [44-46]. He explored various aspects such as RRR, effect of frequency, thermocurrents, presence of magnetically active component etc. His work resulted in a better magnetic shielding of experimental cryostats at our Institute (CEA), and a special care to prevent use of magnetized components. Altogether it allowed the improvement of the mean Q values by a factor 10 , allowing for the first time to get values above $5 \times 10^{10}$ at $2 \mathrm{~K}$ [47]. After that period of improvements to magnetic shielding and cryostat design, field trapping was somewhat forgotten since most of the projects in the 1990s-2000s were more concerned by high gradients. For instance in a project like TTF (Tesla Test Facility, built in Hamburg in the framework of the Tesla collaboration), the temperature regulation was such that most of the time the losses of the cavities were dominated by the BCS component, as the temperatures reached sometimes 2 to $2.2 \mathrm{~K}$ at the end of the cryostats [48]. The implementation of magnetic shielding was sufficient to reduce the residual part in those conditions [49]. Nevertheless, some improved models have been proposed like e.g., [40,50,51]. In [51], Ciovati showed that it was possible to move the hot spots and/or decrease their induced losses by means of a local heating, a scenario well fitting the depinning of flux line with sufficient activation energy. Nevertheless, it was not effective on all hot spots.

Recently this topic became hot again, because in many upcoming projects, especially for $\mathrm{cw}$ machine designs, 
decreasing the cryogenic losses by a factor 2 or 3 leads to significant savings from the point of view of the infrastructure and the operating costs. Many thorough studies have been published recently (see e.g., [41,52-55]), showing the importance of cooling rate, thermal gradient, and the influence of thermocurrents on cavities with their helium cooling jacket (dressed). No complete consensus appears: in some cases high thermal gradients help to expel the field while in some others a small gradient is preferable, differences have been observed between vertical and horizontal cavities, dressed vs bare cavities. It seems that the results are still deeply impacted by some local variations, either in the quality of the niobium or in the variability of the processes applied at each lab.

Flux trapping and imperfect expulsion of the flux at decreasing field is closely related to pinning. Since the details of SRF experiments have been presented elsewhere [56], in the following discussion we will concentrate on the material aspects of pinning, in particular on the occurrence and behavior of specific defects.

\section{TYPICAL FEATURES OF THE NIOBIUM CRYSTAL STRUCTURE}

\section{A. 3D defects (voids, precipitates)}

In niobium like in other superconductors, normal conducting or dielectric precipitates, voids, with dimension larger than $\lambda$, i.e., where the order parameter actually reaches 0 , are the strongest pinning centers. In this case, the total condensation energy can be fully utilized as the pinning energy. The pinning energy of other defects is smaller, since the superconducting parameters vary only slightly due to the electron scattering. Matshushita [4] gives the approximation for elementary pinning force per unit length of flux line normal precipitate: $f_{P} \sim 1.35 \xi \mu_{0} \mathrm{H}_{C^{2}}$. This only includes the condensation energy term, but not magnetic force interaction (which can increase those figures by another $25 \%$ in the case of NC precipitates), or elastic interaction due to the deformation of the lattice... (see [4], Sec. VI for details). For comparison, elementary pinning force for grain boundaries $f_{P} \sim 0.17 \xi \mu_{0} \mathrm{H}_{C^{2}}$. For a normal conducting precipitate in $\mathrm{Nb}$, the elementary pinning force has been evaluated to $f_{P} \sim 9.1 \times 10^{-4} \mathrm{~N} / \mathrm{m}$ [4]. Actually, NbTi alloys can be considered as an example of the role of precipitates in $\mathrm{Nb}$ : it consists of normal conducting Ti precipitates sitting in a matrix of $\mathrm{Nb}$, where only a few of the $\mathrm{Nb}$ atoms are replaced by substitutional Ti. Since $\mathrm{Ti}$ and $\mathrm{Nb}$ have the same diameter, those substitutional Ti atoms do not affect much the matrix, as can be inferred from the fact that $\mathrm{Nb}$ and $\mathrm{NbTi}$ exhibit the same $\mathrm{T}_{C}$ and same $\mathrm{H}_{C}$ at the first order. In fact, only $\xi$ and $\lambda$ (and $\kappa$ ) are strongly affected by the reduction of mean-free path. The pinning force density in $\mathrm{NbTi}$ alloy can reach several $10^{9} \mathrm{~N} / \mathrm{m}^{3}$ at the apparition of the precipitates during heat treatments [57]. The force further increases when drawing is applied to shape $\mathrm{Ti}$ precipitates into ribbons oriented parallel to the flux lines. In the case of normal conducting precipitates, effects like proximity effect or kinetic energy difference also can influence a lot the pinning force, but those effects become appreciable only for very finely divided material with at least one dimension less than $\xi$. Note the following.Elementary pinning force as well as density of pinning force do depend on the applied field and temperature, but not monotonically (scaling laws). The indicated values correspond by convention to the value of the pinning forces at their maximum.

The description of the relationship between elementary pinning force and global pinning force density is very complex [4] and is beyond the scope of this paper.

Inclusions are seldom found in high RRR niobium. Most of the time they result from dust particles being embedded in the smoother $\mathrm{Nb}$ surface during forming processes.

Voids can be found in the heat affected zone when welding are not conducted in clean enough conditions. More rarely, they can also result from the billet fabrication process. Their origin is the light elements segregation in the liquid metal phase during the solidification process.

\section{B. Grain boundaries}

Grain boundaries (GB) and interfaces are 2D defects where the lattice accommodates the differences in atom spacing between two adjoining grains with different orientation (or even different crystal lattice in the case of the oxidemetal interface). This irregular spacing results into lattice distortion that strongly depends on the relative orientations of the grains. GB are usually expected to be effective vortex traps $[4,12]$. Note that only a few relative orientations give rise to high disorder [58]. Certainly, the pinning on GB is most effective when the crystalline disorder around the grain boundary extends in the same order of magnitude as the coherence length $\xi$. This is the case in thin film superconductors, where disorder and grain boundary density are high. Grain boundaries play a role in strong pinning like e.g., high Tc SC or $\mathrm{Nb}_{3} \mathrm{Sn}$ since they have a small $\xi$.

$\mathrm{SRF}$ quality $\mathrm{Nb}$ exhibits equiaxial structure and grains with diameters ranging from $50 \mu \mathrm{m}$ to several $\mathrm{mm}$ in fine grain and even centimeters for large grains. The distortion outside GB does not extend more than a few atomic layers whereas $\xi$ is $\sim 40 \mathrm{~nm}$, or much larger than the distorted region at GB. So most of the grain boundaries in SRF grade $\mathrm{Nb}$ are not expected to provide strong pinning centers [59].

Several experiments demonstrated indeed some weakening of superconductivity at GB. In particular early field penetration can be observed in GB when exposed to magnetic field, forming Josephson vortices with penetration field $\sim 1 / 3$ of the bulk value [12,60-63], the effect being maximum when the field is parallel to the field plane $[3,60,63]$. Grain boundary pinning has been observed and measured on $\mathrm{Nb}[12,64]$, and appears to depend on the grain size. It is believed that large grain material tends to 
exhibit a slightly higher medium field $\mathrm{Q}$ value than fine grain material [24,65-67], because it has less GB. However large grain also exhibits very high quality grain substructure as can be inferred from the high phonon peak observed from thermal conductivity measurements $[68,69]$, and crystalline substructure can as well explain the experimental observations.

Although grain boundaries are obviously areas of weakened superconductivity in bulk $\mathrm{Nb}$, they do not affect particularly SRF behavior. Moreover, although $30 \%$ of the hot spots have been observed close or on the grain boundaries, it was concluded that grain boundaries do not seem to be the main source of hot spots in SRF Nb cavities [59,70-72]. Note that hot spots observed at high field on operating cavities have been attributed to oscillating vortices, which can origin from trapped flux as well as early penetrated vortices $[51,73,74]$. Monocrystalline cavities exhibit qualitatively the same behavior as polycrystalline cavities with similar high field Q-slope before baking $[59,71,75]$. Thus, the reduced pinning behavior observed on large grain cavities cannot be attributed only to the fact that they have fewer grain boundaries (see below).

The elementary pinning force per unit length of flux line $f_{P}$ has been measured in $\mathrm{Nb}$ bicrystals of various relative orientations and it is about $7 \times 10^{-6} \mathrm{~N} / \mathrm{m}$ when the field is aligned parallel to the GB [12], to be compared to values for GB pinning in $\mathrm{Nb}_{3} \mathrm{Sn}$ that range between $6 \times 10^{-5}$ and $10^{-4} \mathrm{~N} / \mathrm{m}$ [64], up to $4 \times 10^{-4} \mathrm{~N} / \mathrm{m}$ for $\mathrm{MgB}_{2}$ [4] and $9.1 \times 10^{-4} \mathrm{~N} / \mathrm{m}$ for a normal conducting precipitate [4]. The difference in the pinning forces between GB and normal conducting precipitates arises mainly from the fact that in GB the main mechanism is electron scattering whereas in normal conducting areas, the dominant mechanism is the savings in condensation energy [4].

Calculating the density of pinning force $\mathrm{Pv}$ from the elementary pinning force $f_{P}$ requires a complex summation problem [76,77]. When one considers grain boundary effects, the direct summation model predicts that the pinning force density is given by [78]

$$
\mathrm{Fp}=f_{P} / \mathrm{D}_{g} \cdot \mathrm{a}_{f},
$$

where $\mathrm{D}_{g}$ is the effective grain size and $\mathrm{a}_{f}$ is the flux line spacing (which varies with $\mathrm{B}$ and $\mathrm{T}$ ). Matsushita estimated these figures for $\mathrm{MgB}_{2}$ and $\mathrm{Nb}_{3} \mathrm{Sn}$ with grain size below $1 \mu \mathrm{m}$, at $\mathrm{B} / \mathrm{B}_{C 2}=0.1$ at $4.2 \mathrm{~K}$ and came up with the maximum achievable pinning force density, respectively $9 \times 10^{10} \mathrm{~N} / \mathrm{m}^{3}$ for $\mathrm{MgB}_{2}$ and $3 \times 10^{10} \mathrm{~N} / \mathrm{m}^{3}$ for $\mathrm{Nb}_{3} \mathrm{Sn}$ [78]. Assuming the same conditions (same $\mathrm{a}_{f}$ ) for $\mathrm{Nb}$ with grain size $\sim 100 \mu \mathrm{m}$, one can estimate $\mathrm{F}_{P}^{N b} \sim 1.75 \times$ $10^{4} \mathrm{~N} / \mathrm{m}^{3}$, i.e., $\sim 6$ orders of magnitude less than with typical SC material used for wire fabrication. For large grain material (grain size $\sim 10 \mathrm{~cm}$ ), $\mathrm{F}_{P}^{N b}$ would reduce further to $\sim 17 \mathrm{~N} / \mathrm{m}^{3}$. So even if grain boundaries exhibit a pinning behavior in $\mathrm{Nb}$, it is not very strong.

\section{Dislocations}

Dislocations are linear defects that are always present in nonperfect crystals (equilibrium concentration depending on temperature). Upon deformation of the material, more dislocations can be emitted to accommodate the strain. In heavily deformed metals, the dislocation density can reach $10^{11}-10^{12} \mathrm{~cm}^{-2}$, and even a very carefully recrystallized material still holds dislocations even at temperatures close to the melting point. Isolated dislocations are expected to have a smaller pinning energy than $2 \mathrm{D}$ defects: the width of a dislocation is small, displacements being comparable with the interatomic distance and confined to a few atoms around the dislocation. Typically the local stress of an individual dislocation reaches $\sim 1 \mathrm{GPa}$ in $2-3$ interatomic distances around the dislocation, but the stress can be multiplied by $\sim 20$ if dislocations pileup occurs [3]. Typically, for $\mathrm{Nb}$ the pinning force per unit length $f_{p}$ is about $10^{-6} \mathrm{~N} / \mathrm{m}$ for a vortex parallel to an isolated single screw dislocation (to be compared to $9.1 \times 10^{-4} \mathrm{~N} / \mathrm{m}$ for a normal conducting precipitate [4]). There are many more dislocations than grain boundaries in typical $\mathrm{Nb}$ used for SRF applications, so the resulting pinning force density (sum of elementary $f_{P}$ ) can become very high.

Moreover, most of the time dislocations form cells where dislocations pile up into "walls" with high stress concentration and high stress gradients whereas the inner cell is nearly defect free, as can be observed on Fig. 4. For heavily deformed material they prefigure future grain or subgrain boundaries that will appear upon recrystallization. Dislocation structure becomes nonuniform in $\mathrm{Nb}$ for very small amounts of deformation (3\%-5\%, equivalent to a density of dislocation $\sim 10^{8}-10^{9} \mathrm{~cm}^{-2}$ ) $[3,79,80]$. Note that the phonon peak disappears from the thermal conductivity curve for the same slight percentage of the deformation, and recovers upon recrystallization [81]. Examples of the effect of recrystallization on the density of dislocation in $\mathrm{Nb}$ can be found e.g., in $[69,82]$.

These types of dislocation cells are clearly 2D defects similar to GB, rather than linear, and their spatial extension is much larger than the distortion around GB, and close to $2 \xi$, which makes them a strong pinning center from the dimensional point of view, in addition to the elastic deformation of the lattice. Therefore, dislocation entanglements are clearly a better candidate for a pinning center in $\mathrm{Nb}$ than GBs. This has several other consequences. Radiofrequency cavities are relatively well recrystallized since they underwent an $800^{\circ} \mathrm{C}$ heat treatment meant to remove hydrogen, but the surface still holds a lot of dislocations. If the damage layer is insufficiently removed, some grains can resist recrystallization $[83,84]$, additionally, the difference of crystalline parameter between oxide layer and the metal underneath produces a lot of strain on the lattice. The density of the pinning force $\mathrm{F}_{P}$ for slightly $(\sim 5 \%)$ deformed samples was found to be $\sim 2-6 \times 10^{5} \mathrm{~N} / \mathrm{m}^{3}$ [3,85-87], i.e., about 10 to 25 times higher the density of the pinning force 
$\mathrm{F}_{P}$ due to grain boundaries in $\mathrm{Nb}$ with $100 \mu \mathrm{m}$ diameter grains, and orders of magnitude larger than for the large grain material.

Dislocations do not appear at random locations; they move along slip planes and within the slip planes, there are preferred crystallographic directions for dislocation movement (slip directions), typical of a $\mathrm{BCC}^{2}$ lattice for $\mathrm{Nb}$ $[9,88,89]$. This means that during forming of a polycrystalline material, not all grains deform the same way depending on their initial orientation (this phenomenon is at the origin of the apparition of orange peel when forming metals). Because of an "unfavorable" orientation, some grains will see their density of dislocations increase a lot, and they might end with a specific texture that is hard to recover even after full recrystallization. This aspect is particularly strong after cold rolling where the surface texture has shown to be resistant to recrystallization [83]. Materials with severe near surface deformation exhibit a thin surface layer able to support current densities up to 2 orders of magnitude higher than the bulk values [3], which is a good indication of surface pinning. This scenario explains very well the patchy nature of hot spots as described by Romanenko [90] (see discussion in Sec. V), but also why even after recrystallization at $800^{\circ} \mathrm{C}$ some grains retain a high density of dislocations.

For instance, Hering [91] found a density of dislocation as low as $10^{6}-10^{7} \mathrm{~cm}^{-2}$ in monocrystalline, RRR 3000, well-recrystallized niobium. He evidenced pinning with a decoration technique showing the deformation of the Abrikosov lattice combined with transmission electron microcopy (TEM) inspection. The recrystallized material tested showed weak pinning occurred and the vortices exhibit a regular Abrikosov lattice. The same material after cold work exhibits strong pinning, with vortex lattice destroyed at a large scale. Short scale ordering can still be observed inside the dislocation cells, but stops at the cell walls. All vortice lines at a short distance from the wall get attracted there. Similar effects have been observed in $[14,82]$. In [14], Sathanam draws a correlation between $\mathrm{J}_{C}$ (which is proportional to the pinning force $\mathrm{F}_{P}$ ) and the mean size of dislocation cells. He found that $\mathrm{F}_{P}$ is maximum for dislocation cells about $100 \mathrm{~nm}$, which is precisely the same order of magnitude as $2 \xi$.

\section{Dislocation cells and diffusion}

Dislocations are expected to strongly influence atomic diffusion during recrystallization or doping experiments. For instance, the existence of interstitial atoms diffusing slower than the dislocations can slow the recrystallization process because of the drag force they exert on the moving dislocation. Inversely diffusing atoms will tend to segregate on dislocations giving rise to nonuniform distribution of interstitials. The temperature at which dislocations and interstitials move $\sim$ at the same rate is

${ }^{2}$ BCC: Body Centered Cubic. around $200{ }^{\circ} \mathrm{C}-350{ }^{\circ} \mathrm{C}$ for oxygen and $400{ }^{\circ} \mathrm{C}-500{ }^{\circ} \mathrm{C}$ for carbon and nitrogen, the exact temperature depends on the niobium purity [92].

Concerning doping experiments with nitrogen, it is quite interesting that Martinello found that for cavities a maximum trapped flux sensitivity was also centered on the same $100 \mathrm{~nm}$ value for mean-free path [93,94]. On the other hand, similar experiments performed at Cornell showed a maximum trapped flux sensitivity for niobium with a mean-free path centered around $8 \mathrm{~nm}$ [50], showing that nonreproducible elements are involved in the fabrication of the cavities from lab to lab. Such a short mean-free path seems to indicate that the $\mathrm{Nb}$ of the Cornell cavities is rather dominated by point defects (see the next section) while the cavities from FNAL were dominated by dislocation cells, probably remaining traces from surface damage.

Figure 5 (right) shows EBSD image quality (IQ) of an electropolishing (EP) niobium sample before and after baking (EBSD). IQ is a normalized index calculated by comparing the actual diffraction pattern to an ideal one. IQ is influenced by all types of dislocations (geometrically necessary as well as statistically stored ones), vacancies and impurities. It is an excellent diagnostic of crystalline quality. On this picture one can see that some grains are darker than others (i.e., more "defective"), and that baking affects only some of them. The overall effect is not necessarily an improvement since baking seems to reduce dislocation density [95] but also increases interstitial atoms distribution [96,97]. Etching figures as shown on Fig. 5 (left) are a way to visualize dislocation cells emerging at the surface of the crystal.

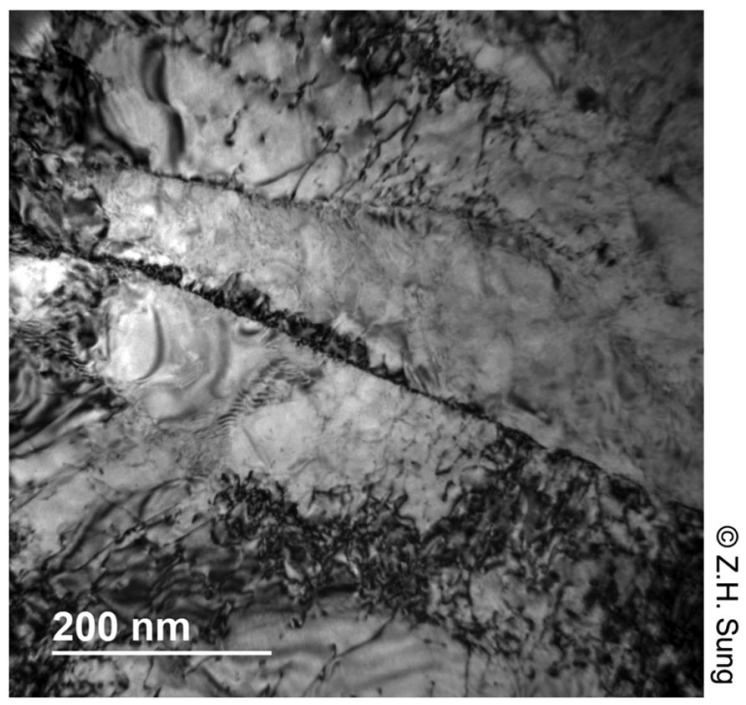

FIG. 4. TEM picture from $5 \%$ tensile stretched $\mathrm{Nb}$. The original picture was reproduced with the kind authorization from $\mathrm{Z}$. H. Sung. One can distinctly observe the spatial extension of dislocation bundles (dark contrast lines). For other pictures of dislocation cells see [82] or [98]. 

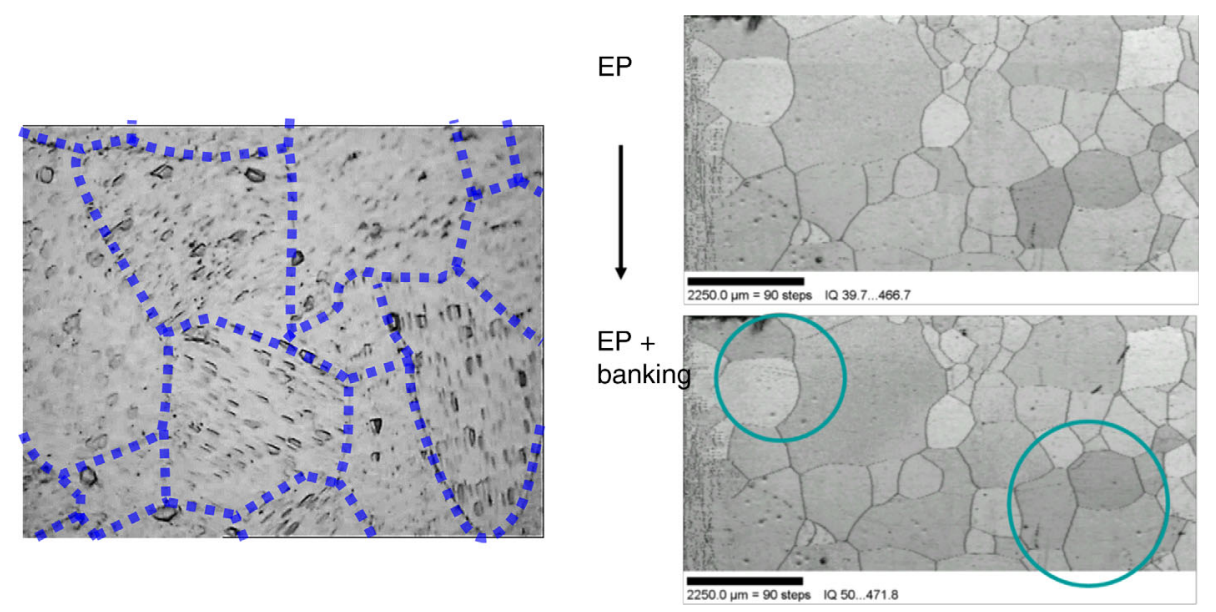

FIG. 5. Left: Etching figures revealing dislocation arrays on as-received $\mathrm{Nb}$ sheet. The blue dotted line marks grain boundary locations. Note how the etching figures obviously depend on crystalline orientation. Right: Crystalline image quality index (see the text for explanation) as determined by EBSD. The darker areas show grains where the diffraction diagrams are distorted compared to the expected diagram from a "perfect crystal." Note that not all the grains have the same relative quality, and that baking does not necessarily improve the local IQ (only $12 \%-13 \%$ of the grains are affected by baking).

\section{Interstitials and vacancies}

Point defects can either be substitutional or interstitial atoms and vacancies. As stated before, the pinning potential of point defects is expected to be lower than planar or linear defects, and their effect become sensible only when present in high density. For instance, it was demonstrated that oxygen vacancies are the main pinning defect in Bi-2212 [20]. Point defect created by irradiation is known to provide collective pinning defects in a large variety of superconductors [4]. Point defect constitutes a typical example of collective pinning due a large density of weak pinning centers, as treated by Larkin and Ovchinnikov [76] for $\lambda_{L}>\mathrm{a}_{0}, \mathrm{a}_{0}$ being the diameter of the weak pinning center. Of course in the case of $\mathrm{Nb}$, where $\xi$ is large compared to the diameter of the distorted lattice volume due to point defect, the volume effect in $\xi / \mathrm{a}_{0}$ is expected to be low. However, Thuneberg et al. in [99] have shown that in the case of defects with very small radius $\left(\mathrm{a}_{0}\right)$, an additional quasiparticle scattering mechanism leads to large elementary pinning energies that outweighs the traditional volume pinning force in $\xi / \mathrm{a}_{0}$.

In $\mathrm{SRF} \mathrm{Nb}$ substitutional atoms are not likely to play a major role: they are minor impurities, and the most abundant of them, $\mathrm{Ta}$, has the same diameter as $\mathrm{Nb}$; it is perfectly miscible in the $\mathrm{Nb}$ lattice and does not perturb the lattice much. Interstitial atoms and vacancies on the other hand seem to play a paramount role in $\mathrm{Nb}$ performance.

In [13], Ullmaier et al. studied the influence of Frenkel pairs (one self-interstitial atom + one vacancy) created by irradiation below $\mathrm{T}_{C}$ in a nearly perfect $\mathrm{Nb}$ wire (reversible magnetization curve). In such conditions they probed defects with relative distance between 20 and $4.5 \mathrm{~nm}$, i.e., between 20 and 500 defects per $(\xi)^{3}=10^{-16} \mathrm{~cm}^{3}$. As expected, the maximum pinning force depends on the defect density, but not monotonically, which suggests the existence of a fluctuation in the defect density responsible for pinning. Indeed, one expects defects to require more energy to be created in e.g., close packed crystalline directions, so irradiation defects (as well as other kinds of punctual defects or diffusing species) have no reason to be distributed uniformly at the small scale. About the physical origin of the pinning interaction, this paper points out the local modification of the lattice elastic energy. Definitely, $\Delta \mathrm{Y} / \mathrm{Y}$, the relative elastic modulus difference between normal and SC states, is known to decrease with mean-free path [13]. Elastic strain of the crystal lattice then interacts with the elastic strain of the vortices lattice. Large quantities of interstitial atoms are known to compress elastically the crystal lattice and influence its SC properties. One wellknown effect is e.g., the slight enhancement of $\mathrm{T}_{C}$ in doped and/or impure $\mathrm{Nb}$ [100] or in strained thin films, see e.g., [101]. In addition, literature seems to indicate that interstitials play an important role in dislocation behavior upon performing annealing: although pinning can be observed even in very pure samples, it is higher if niobium with some interstitial content is annealed just after a deformation step. Even small amounts of interstitial impurities seem to affect the annealed structure of cold worked niobium [3,4].

Vacancies also seem to play a paramount role close to the surface. Two authors [102,103] point out the augmentation of vacancies concentration close to the surface (50$100 \mathrm{~nm})$ after baking $\left(120^{\circ} \mathrm{C}, 48 \mathrm{~h}\right)$ of chemically etched $\left(\mathrm{BCP}^{3}\right)$ surfaces. Results seem a little more ambiguous concerning the EP surface. Both authors mention the existence of vacancies-H coupling, but their interpretation is radically different: Visentin [103] points out the dissociation of those V-H compounds, while Romanenko [102] proposes the creation of many new ones via vacancies

\footnotetext{
${ }^{3}$ BCP: Buffered Chemical Polishing.
} 
incorporation from the surface. We think that the first scenario is better supported experimentally as will be discussed below in Sec. IV A (Segregations due to crystalline defects). Romanenko also proposed that this release of vacancies favors dislocation movements and is at the origin of the decrease of misorientation angles after baking [104].

\section{E. Conclusion about flux trapping sensitivity}

A vortex typically interacts with either clusters of a few pinning defects separated by about the coherence length [105] or by evenly distributed weak pinning centers at high density. All data found in literature points out an effect of fluctuations at the scale of some $\mathrm{nm}$ to some $10 \mathrm{~s}$ of $\mathrm{nm}$ on $\mathrm{SC}$ parameters. Among the main features liable to be at the origin of flux trapping in SRF cavities, dislocation tangles and/or dislocation cells seem to be the most efficient mechanism since they exist in large number even in well recrystallized niobium. Obviously large quantities of interstitials can play an additional role, as it has been demonstrated with e.g., doping experiments [50,93]. It is very unlikely that we master yet SRF superconductors, bulk $\mathrm{Nb}$ as well as thin films $\mathrm{Nb}$ and alternative $\mathrm{SC}$, at this scale.

Recent work done on cavity doping [56] also points out the influence of interstitials in the flux trapping behavior of $\mathrm{Nb}$ cavities. In the next chapter, we will deal with their possible effect on the prevention of hydride precipitation.

\section{HYDRIDES}

Hydrides have been identified in the early 1990s as a source of increased rf surface dissipation upon slow cooldowns [106-112]. Although the bulk concentration was less than 0.01 at. \%, precipitation of hydrides was evidenced upon slow cooldown (rate $<1 \mathrm{~K} / \mathrm{min}$ ) or when high RRR cavities were allowed to stay between 75-150 K (with a saturation of the effect after 3 hours [112]). The quality factor $\mathrm{Q}_{0}$ value of slow cooled cavities can lose up to 3 orders of magnitude, and $\mathrm{R}_{\mathrm{BCS}}$ as well as the residual resistance $\mathrm{R}_{0}$ are affected [108]. The incorporation of $H$ happens principally during etching, but it is a collateral effect resulting from the absence of the oxide, which is an effective diffusion barrier to atomic $\mathrm{H}$ [110]. In the same way, niobium with no oxide or nonpassivated oxide is also very sensitive to $\mathrm{H}$ : annealed niobium if kept under vacuum for a long time, niobium dipped in oxide removing solutions $(\mathrm{NaOH}, \mathrm{HCl}, \mathrm{HF}, \ldots)$, and even niobium in contact with boiling water ${ }^{4}$ [113]. Hydrogen is known to segregate close to dislocations and form so-called Cottrell

\footnotetext{
${ }^{4}$ Note that typically degreasing is conducted in slightly basic detergent solution at around $50^{\circ}-60^{\circ}$. A survey of this step was never closely documented, although it could be at the origin of some of the $\mathrm{H}$ pollution, basic detergent solution being depassivating and water at high temperature known to be harmful.
}

clouds [114-117], due to the elastic interaction phenomena described in Sec. III B and below.

\section{A. Segregation due to crystalline defects}

The apparition of hydrides at such low bulk concentration is made possible by the fact that hydrogen, although it is very mobile in $\mathrm{Nb}$, tends to segregate near the surface. This type of segregation is typical of pure metals: indeed hydrogen tends to interact with defects that elastically deform the crystal lattice, the interaction energy increasing from a punctual defect (interstitial atom, vacancy), to a linear defect (dislocation), and to a 2D defect (interfaces like GB, and mostly metal oxide interface). In a sense the defects that tend to pin vortices are also the ones that tend to trap hydrogen atoms, because of the elastic strain they produce on the lattice ([107] and references therein).

In a pure metal most of the defects left are concentrated close to the surface, more exactly to the metal-oxide interface. Measurements show that just underneath the oxide layer the surface concentration of $\mathrm{H}$ can reach some 0.1 at. $\%$ to some at.\% [107,111]. The oxide-metal interface is a complex area with a lot of strain and dislocations necessary to accommodate the difference in crystalline parameters. Moreover, the surface also keeps traces from the damage layer, mainly produced by rolling and deep drawing of the $\mathrm{Nb}$ sheets and even after heavy BCP or EP some grains with a higher density of dislocations still remain [118]. Romanenko showed on cavity cutouts a correlation between hot spots and density of hydrides as well as higher misorientation angles [104]. Hydrides have also been evidenced by Raman spectroscopy: characteristic vibrational bands from $\mathrm{NbH}$ and $\mathrm{NbH}_{2}$ have been observed on cold as well as hot spots [119], but their density seems to be higher on hot spots. The same trend has been observed by TEM [120].

Note that some of the hydrogen can also be trapped by vacancies. Two situations may occur: in a single vacancy up to six $\mathrm{H}$ atoms can be trapped, and there are evidences that more $\mathrm{H}$ tends to gather in the vicinity, providing nucleation sites for hydrides [121]. In the presence of interstitials atoms like $\mathrm{O}, \mathrm{N}, \mathrm{C}$, those atoms will occupy preferentially the vacancy: each oxygen, nitrogen or carbon impurity traps only a single hydrogen atom under formation of oxygen-hydrogen, nitrogen-hydrogen or carbon-hydrogen pairs, where the trapped hydrogen occupies interstitial trap sites in the neighborhood of the trapping impurity [122125]. Specific interaction between interstitial oxygen and hydrogen has been thoroughly studied in niobium $[117,123,124,126-130]$ as it presents a textbook example of a two-well tunneling system.

There is direct evidence that hydride precipitation is inhibited due to trapping at $\mathrm{O}, \mathrm{N}$ or $\mathrm{C}$ interstitials $[122,131]$. Moderate vacuum baking (typically $120^{\circ} \mathrm{C}$, $48 \mathrm{~h}$ ) is known to break the interstitial-hydrogen bond $[116,125,129]$. It has also been proven that the near surface is enriched with interstitials, mainly $\mathrm{O}[83,107]$, and that 
baking tends to increase the $\mathrm{O}$ content in the near surface $[97,132]$. We can thus infer a scenario explaining in the same time the baking effect and doping experiments: if the interstitial content near the surface ( $\sim r f$ penetration depth) is increased, the interaction of $\mathrm{H}$ with those interstitials will prevent accumulation of hydrogen and reduce the apparition of hydrides. Recent TEM experiments show a decrease in hydride formation after $120^{\circ} \mathrm{C}$ baking [120]. Hydrides being poor superconductors, they can promote early penetration of a vortex, thus by reducing their presence, one can reach higher fields without early nucleation of vortex, which is basically what is observed after baking. The initial absence of vortex is sustained by $\mu$-SR experiment showing a strong correlation between the onset of the HFQS rf losses and the entry of magnetic flux on masked samples ${ }^{5}$ [31]. Another indication of the good crystalline quality of "cold spots" vs hot spots is given by tunneling $\mathrm{PCT}^{6}$ experiment: cold spots exhibit a nearly ideal BCS behavior, while hot spots show enlarged density of state, localized energy level inside the gap and other features relevant from inelastic pair breaking [133].

\section{B. Heat treatments: Necessary but not fully efficient}

Heat treatments (UHV, typically $800^{\circ} \mathrm{C}, 2 \mathrm{~h}$, but also $600^{\circ} \mathrm{C} \mathrm{5-10} \mathrm{h)} \mathrm{allow} \mathrm{to} \mathrm{get} \mathrm{rid} \mathrm{of} \mathrm{most} \mathrm{of} \mathrm{the} \mathrm{hydrogen}$ causing heavy Q-disease, i.e., the strong decrease in quality factor that appears at a few $\mathrm{MV} / \mathrm{m}$ if the cavity is maintained a few hours around $100 \mathrm{~K}$. From the thermodynamic point of view, $\mathrm{H}$ is meant to degas partially, the kinetic being controlled by diffusion [134]. After $800^{\circ} \mathrm{C}$, 2 hours, some hydrogen keeps trapped inside the $\mathrm{Nb}$ matrix. The effect of annealing is multiple: as mentioned before, it will dissociate vacancies-H bonds; it will render the distribution of $\mathrm{H}$ more or less uniform in the material and in the same time, will provide some restauration/ recrystallization of the material. Upon cooling, some hydrogen uptake happens as the niobium is not protected by its oxide layer anymore (Sievert's law), and the remaining hydrogen tends to segregate back toward the surface [107,135]. Nevertheless, annealed cavities are much less sensitive to hydrogen uptake during subsequent chemical polishing. This is probably related to the fact that the annealing allows the lattice to recrystallize and/or recover, and thus the quantity of crystalline defects, in particular dislocations are tremendously reduced, leaving less preferential sites for segregation [136,137]. One argument that supports further the recrystallization hypothesis is that the couples "times + temperature" observed to remove Q-disease are typically those known for recrystallization: the relationship between time $\left(t_{R}\right)$ and temperature $\left(T_{R}\right)$

\footnotetext{
${ }^{5}$ An annular mask allows to center the measurement where no early penetration of vortex due to edge effects/demagnetization factor occurs.

${ }^{6}$ PCT: Point Contact Tunneling.
}

to achieve a given recrystallization state $\mathrm{R}$ is in the form of [138]

$$
\log t_{R}=\mathrm{A}+\mathrm{B}\left(\frac{1}{T_{R}}\right)
$$

where time is in hours and temperature in Kelvins. A and B depend on the purity and deformation state prior to annealing.

For a typical $\mathrm{Nb}$ batch, it was determined that the law was [83], but it probably needs to be estimated precisely for each batch of material:

$$
\log t_{R}=25861 \cdot\left(\frac{1}{T_{R}}\right)-22.28
$$

Nevertheless, even if heat treatment gets rid of heavy Q-disease, experimental facts described in the previous sections indicate that it is not possible to eliminate all the crystalline defects, and some hydrogen remains. There are some indications that different kinds of hydrides form depending on whether the $\mathrm{H}$ content is low or high and on the crystalline state of the surface [136].

\section{FLUX TRAPPING VS FLUX EARLY ENTRY}

Hotspots are attributed to the presence of vortices [51,73], but these vortices can have two possible origins: initially a trapped flux line, or early flux penetration when increasing $\mathrm{rf}$ field. As stated before any defect with depleted superconductivity is liable to promote early vortex entry as well as pinning behavior. The pinning force also depends on field, and it is possible that the predominant kind of defect is not the same as in the case of remnant flux trapping (compared to early penetration of the rf field). There are several experimental evidences that support each phenomenon, and they probably coexist in many cases.

The patchy nature of hot spots has been established quite early [47], but it took a thorough work from Romanenko on cavity cutouts for a better understanding to appear $[95,139]$. Romanenko and collaborators worked on samples cut out of cavities (small grain and large grain, processed by BCP and EP) and compared cold and hot spots from the structural, morphological and chemical point of view $[3,4,16,17]$. Recently these results have been completed with magnetic characterization that highlights some aspects of vortice pinning [31,140].

The main difference between cold and hot spots was observed by an electron diffraction technique that probes the first $\sim 100 \mathrm{~nm}$ of the sample (EBSD, also used for orientation imaging). Indexation of the diffraction pattern gives the exact orientation of each grain, but can also provide a means to evaluate local misorientation and indirectly dislocation density. Romanenko observed that hot spots tend to exhibit higher misorientation angles and 
TABLE I. $\quad$ Trapped vortices vs early penetration: Experimental arguments [25,31,32,83,140,144-146].

Trapped vortices

- $\mu$ SR experiments (penetration field corrected from geometrical demagnetization effects): shows different field penetration in function of the crystalline state (etched, formed, $1400^{\circ} \mathrm{C}$ annealed)

- Irreversibility observed in dc magnetization curve as explained in Sec. II A

- Flux jumps on some magnetization curves (NB. Flux jumps are more often observed on damaged or polluted surfaces).

- 0 magnetic moment on field cooled dc experiments on standard SRF Nb, indicating 0 flux expulsion [147]

- Depinning frequency is very low for bulk well recrystallized monocrystalline $\mathrm{Nb}\left(10^{4} \mathrm{~Hz}\right)$ : trapped flux is then expected to be mobile in rf field and produce dissipation
Early penetration

- Morphologic defects are often found at the quench site (step, pit): in this case, the local field is higher than the applied field. It can also be considered as having a defect with locally lower $\mathrm{B}_{C 1}$

- Large hydride precipitates provide areas with both lower $\mathrm{H}_{C 1} / \mathrm{T}_{C}$, but also deform the surface and produce morphologic defects [136]

- Point contact tunneling shows degraded SC properties in hot spot (inelastic quasiparticle scattering: broadened DOS, a finite zero bias conductance and/or zero bias peak, lower gap...): could promote early penetration thus higher dislocation density. ${ }^{7} \mathrm{He}$ also found that $\mathrm{Nb}$ cutout samples, after a metallographic mechanical polishing, exhibit characteristic features of hydride precipitates (snowflake like features highly visible on cold SEM pictures), and that the density of hydrides is higher on hot spots than it is on cold spots [141,142]. A similar hydride visualization technique has also been used in [143]. It is obvious that high dislocation density and the presence of high concentration of hydrides are related.

As stated earlier, hot spots can either be attributed to trapped flux line at cooldown of cavities or to early flux penetration with increasing rf field. In Table I we list the experimental arguments in favor of one or the other hypothesis. It is highly probable that both phenomena coexist.

It is interesting to note that $\mathrm{Nb}$ thin films show common properties with bulk niobium hotspots, in particular the existence of subgap states as measured with point contact tunneling [140], since one knows that thin films usually exhibit high crystalline defect density: strained lattice with higher $\mathrm{T}_{C}$, very small grains, high density of displaced and foreign atoms. Similar problems are also expected to be encountered for alternative $\mathrm{SRF}$ superconductors produced in the form of thin films like $\mathrm{Nb}_{3} \mathrm{Sn}, \mathrm{MgB}_{2}$, multilayers.

\section{CONCLUSION}

It is interesting to note that the crystalline state dominates two of the most harmful phenomena limiting SRF performance: flux trapping and early flux penetration due to hydrides. Obviously, dislocation cells remaining from the damage layer are the main source of variability, and some

\footnotetext{
${ }^{7}$ Note that with the misorientation technique used here, one is sensitive only to the geometrically necessary dislocation resulting from lattice deformation. It does not give information on the statistical crystalline disorder (equilibrium density of dislocation, vacancies, impurities,...).
}

efforts should be made to better understand how reproducible it is from each batch, and its effective removal.

Even if one cannot get a perfect surface in realistic conditions there is still room for optimization of the cavity preparation process. Dealing with bulk $\mathrm{Nb}$, during the fabrication, several aspects are not followed closely enough to get reproducible quality of niobium within the SRF depth of interest, i.e., $500 \mathrm{~nm}$ [83].

Recrystallization: During Nb sheet fabrication, several steps of heavy deformation + recrystallization stages occur. First, depending on the desired thickness, the deformation ratio is not exactly the same for all the sheets. Second, depending on their position in the oven, not all sheets undergo the recrystallization treatment at the same exact temperature. It follows that the dislocations distribution in the material can vary a lot within the same batch, and even more from batch to batch or vendor to vendor.

Surface damage due to rolling, and specifically skin pass ${ }^{8}$ are known to affect a deeper thickness than the $150 \mu \mathrm{m}$ usually chemically removed from the cavity surface. As pickling of the sheets is generally applied at the vendor and removes an unknown amount of damage layer, the actual damage thickness to be removed is not known with precision. This aspect also contributes to batch to batch variation.

Forming and welding of cavities also contributes to additional strain. In particular, welding is somehow equivalent to a heat treatment and produces some recovering, but if not conducted correctly, it can also give rise to local thermal stresses in the heat affected zone because of the huge thermal gradient caused by EB welding. This remaining stress can be at the origin of local stress corrosion and the apparition of pits during subsequent etching.

\footnotetext{
${ }^{8}$ Skin pass is a finishing step of the rolling procedure. After the rolling, usually the metal sheet is not flat; so the sheet is conducted through a series of small rolling mill to achieve the desired flatness.
} 
Electropolishing (EP) and/or centrifugal barrel polishing of cavities have a beneficial effect as it allows removing most of the damaged layer, but here again, some grains more defective than the adjoining ones may persist if not enough material is removed.

The baking and heat treatments usually applied after heavy EP/BCP of cavities have a beneficial effect as they allow recovering a large portion of crystal defects, but since there is not a universal recipe among different labs, it is also a source of nonreproducibility. Moreover, if these treatments seem to be beneficial in terms of crystal structure, they tend to increase the interstitial content, and might replace a strong localized pinning with multiple weak pinning centers (magnetic to volume pinning). A similar effect can be expected in case of $\mathrm{N}$-doping or $\mathrm{N}$ infusion. Determining the proper balance between various treatments depending in the desired performance will be for sure the next challenge in SRF.

The crystallographic quality required for SRF application is beyond the reach of traditional metallurgical characterization (tensile curves, micrographs, hardness, ...). Ultimately it would be beneficial to systematically assess the surface state of representative coupons (which underwent a similar process as the whole cavity fabrication) with advanced techniques (like EBSD QI, PCT, $\mu \mathrm{SR}$, etc.) to have a better understanding and predictability of the material batch to batch, and cavity to cavity. Even if it is expensive, cutting actual cavities has proven a valuable source of information and some provisions should be made to continue in this direction.

[1] G. Seibold, L. Benfatto, and C. Castellani, Application of the Mattis-Bardeen theory in strongly disordered superconductors, Phys. Rev. B 96, 144507 (2017).

[2] D. C. Larbalestier, Basics on SC for accelerator builder, CAS Erice, 2013, http://cas.web.cern.ch/cas/Erice-2013/ Lectures/Ericelectures.htm.

[3] A. Campbell and J. Evetts, Flux vortices and transport currents in type II superconductors, Adv. Phys. 21, 199 (1972).

[4] T. Matsushita, Flux Pinning in Superconductors (Springer, New York, 2007), Vol. 164.

[5] P.-G. De Gennes and P. A. Pincus, Superconductivity of Metals and Alloys (W.A. Benjamin, New York, 1966), Vol. 86.

[6] A. Gurevich and T. Kubo, Surface impedance and optimum surface resistance of a superconductor with an imperfect surface, Phys. Rev. B 96, 184515 (2017).

[7] A. Gurevich, Theory of rf superconductivity for resonant cavities, Supercond. Sci. Technol. 30, 034004 (2017).

[8] D. B. Liarte, S. Posen, M. K. Transtrum, G. Catelani, M. Liepe, and J.P. Sethna, Theoretical estimates of maximum fields in superconducting resonant radio frequency cavities: Stability theory, disorder, and laminates, Supercond. Sci. Technol. 30, 033002 (2017).
[9] R. J. Tilley, Defects in Solids (John Wiley \& Sons, New York, 2008), Vol. 4.

[10] U. Messerschmidt, Dislocation Dynamics During Plastic Deformation (Springer Science \& Business Media, Berlin, 2010), Vol. 129, DOI: 10.1007/978-3-642-03177-9.

[11] J. R. Clem, A model for flux pinning in superconductors, Low Temp. Phys. 13, 102 (1972).

[12] A. D. Gupta et al., Flux pinning by symmetrical grain boundaries in niobium bicrystals, Philos. Mag. 38, 367 (1978).

[13] H. Ullmaier, K. Papastaikoudis, S. Takács, and W. Schilling, Pinning of flux lines in superconducting niobium due to point defects, Phys. Status Solidi (b) 41, 671 (1970).

[14] A. Santhanam, Flux-pinning in multifilament niobium by dislocation cell boundaries, J. Mater. Sci. 11, 1099 (1976).

[15] J. Gail, M. Mück, and C. Heiden, Pinning and depinning of single vortices in niobium thin film dc superconducting quantum interference devices, Appl. Phys. Lett. 73, 2663 (1998).

[16] W. R. Hudson, R. J. Jirberg, and J. Simmons, Flux pinning in superconducting niobium thin films, Phys. Lett. 21, 493 (1966).

[17] G. Alers and D. Waldorf, Variation of the elastic moduli at the superconducting transition, Phys. Rev. Lett. 6, 677 (1961).

[18] C. Bean and J. Livingston, Surface barrier in type-II superconductors, Phys. Rev. Lett. 12, 14 (1964).

[19] P. Lee and D. Larbalestier, Determination of the flux pinning force of $\alpha$-Ti ribbons in $\mathrm{Nb} 46.5 \mathrm{wt} \%$ Ti produced by heat treatments of varying temperature, duration and frequency, J. Mater. Sci. 23, 3951 (1988).

[20] T. W. Li, A. A. Menovsky, J. J. M. Franse, and P. H. Kes, Flux pinning in Bi-2212 single crystals with various oxygen contents, Physica (Amsterdam) 257C, 179 (1996).

[21] A. C. Rose-Innes, Introduction to Superconductivity (Elsevier, New York, 2012), Vol. 6.

[22] M. Bahte, F. Herrmann, and P. Schmuser, Magnetization and susceptibility measurements on niobium samples for cavity production, Part. Accel. 60, 121 (1998).

[23] W. Beall Jr. and R. Meyerhoff, AC energy losses above and below $\mathrm{Hc} 1$ in niobium and niobium-25 At.\% zirconium, J. Appl. Phys. 40, 2052 (1969).

[24] P. Kneisel et al., Review of ingot niobium as a material for superconducting radiofrequency accelerating cavities, Nucl. Instrum. Methods Phys. Res., Sect. A 744, 045008 (2015).

[25] T. Junginger et al., Field of first magnetic flux entry and pinning strength of superconductors for $\mathrm{rf}$ application measured with muon spin rotation, Phys. Rev. Accel. Beams 21, 032002 (2018).

[26] C. P. Bean, Magnetization of hard superconductors, Phys. Rev. Lett. 8, 250 (1962).

[27] G. Van Gurp and D. Van Ooijen, The influence of dislocations on superconductivity, J. Phys. Colloques 27, C3-51 (1966).

[28] A. Pautrat, J. Scola, C. Goupil, Ch. Simon, C. Villard, B. Domengès, Y. Simon, C. Guilpin, and L. Méchin, Quantitative analysis of the critical current due to vortex 
pinning by surface corrugation, Phys. Rev. B 69, 224504 (2004).

[29] V. A. Khokhlov, A. I. Kosse, Yu. E. Kuzovlev, G. G. Levchenko, Yu. V. Medvedev, A. Yu. Prokhorov, P. Mikheenko, R. Chakalov, and C. M. Muirhead, Surface pinning as origin of high critical current in superconducting films, Supercond. Sci. Technol. 17, S520 (2004).

[30] E. H. Brandt, Superconductors in realistic geometries: Geometric edge barrier versus pinning, Physica (Amsterdam) 332C, 99 (2000).

[31] A. Grassellino, C. Beard, P. Kolb, R. Laxdal, N. S. Lockyer, D. Longuevergne, and J. E. Sonier, Muon spin rotation studies of niobium for superconducting $\mathrm{rf}$ applications, Phys. Rev. ST Accel. Beams 16, 062002 (2013).

[32] N. Lütke-Entrup, B. Plaçais, P. Mathieu, and Y. Simon, Depinning transition in type-II superconductors, Phys. Rev. Lett. 79, 2538 (1997).

[33] V. Shklovskij and O. Dobrovolskiy, Determination of coordinate dependence of a pinning potential from a microwave experiment with vortices, Low Temp. Phys. 39, 120 (2013).

[34] M. Checchin, M. Martinello, A. Grassellino, S. Aderhold, S. K. Chandrasekaran, O. S. Melnychuk, S. Posen, A. Romanenko, and D. A. Sergatskov, Frequency dependence of trapped flux sensitivity in SRF cavities, Appl. Phys. Lett. 112, 072601 (2018).

[35] J. I. Gittleman et al., Nonlinear reactance of superconducting films, Phys. Rev. 137, A527 (1965).

[36] J. I. Gittleman and B. Rosenblum, The pinning potential and high-frequency studies of type-II superconductors, J. Appl. Phys. 39, 2617 (1968).

[37] M. S. Grbić, D. Janjušević, M. Požek, A. Dulčić, and T. Wagner, Microwave study of magnetic field penetration parallel to thin niobium films, Physica (Amsterdam) 460C, 1293 (2007).

[38] D. Janjušević, M. S. Grbić, M. Požek, A. Dulčić, D. Paar, B. Nebendahl, and T. Wagner, Microwave response of thin niobium films under perpendicular static magnetic fields, Phys. Rev. B 74, 104501 (2006).

[39] V. Palmieri, rf losses due to incomplete MeissnerOchsenfeld effect: Difference between bulk $\mathrm{Nb}$ and $\mathrm{Nb} / \mathrm{Cu}$, 4th International Workshop on Thin films applied to superconducting RF and new ideas (TFSRF 2010), Padova, Italy, 2010, http://www.slideshare.net/ thinfilmsworkshop/palmieri-rf-losses-trapped-flux.

[40] A. Gurevich and G. Ciovati, Dynamics of vortex penetration, jumpwise instabilities, and nonlinear surface resistance of type-II superconductors in strong rf fields, Phys. Rev. B 77, 104501 (2008).

[41] S. Aull and J. Knobloch, Depinning of trapped magnetic flux in bulk niobium SRF cavities, arXiv:1507.04105.

[42] J. Turneaure and I. Weissman, Microwave surface resistance of superconducting niobium, J. Appl. Phys. 39, 4417 (1968).

[43] C. Benvenutii, Magnetic flux trapping in superconducing $\mathrm{Nb}$, Proceedings of the 8th Workshop on RF Superconductivity, Abano Terme (Padova), Italy, 1997 (JACoW, Geneva, Switzerland, 1997), https://accelconf .web.cern.ch/AccelConf/SRF97/papers/srf97b05.pdf.
[44] C. Vallet et al., Flux trapping in superconducting cavities, in Proceedings of 3rd European Particle Accelerator Conference (EPAC 1992), Berlin, Germany, 1992 (JACoW, Geneva, Switzerland, 1992), http://accelconf.web .cern.ch/accelconf/e92/PDF/EPAC1992_1295.pdf.

[45] C. Vallet, M. Bolore, and B. Bonin, Residual rf surface resistance due to trapped magnetic flux, CEA internal Report No. DAPNIA-SEA-93-15, available at http:// inspirehep.net/record/360831/.

[46] C. J. Vallet, Etude de la dissipation dans les supraconducteurs en régime haute-fréquence Ph.D. thesis, Université de Lyon 1, Lyon, France, 1994.

[47] H. Safa, High gradients in SRF cavities, in SRF cavities Proceedings of the 8th Workshop on RF Superconductivity, Abano Terme (Padova), Italy, 1997 (JACoW, Geneva, Switzerland, 1997), http://accelconf.web.cern .ch/AccelConf/SRF97/papers/srf97d03.pdf.

[48] C. Pagani (private communication).

[49] M. Boloré et al., Magnetic shielding of the TESLA TTF superconducting cavities, TESLA-Report No. 1994-23, 1994, http://cds.cern.ch/record/1113540.

[50] D. Gonnella, J. Kaufman, and M. Liepe, Impact of nitrogen doping of niobium superconducting cavities on the sensitivity of surface resistance to trapped magnetic flux, J. Appl. Phys. 119, 073904 (2016).

[51] G. Ciovati and A. Gurevich, Evidence of high-field radio-frequency hot spots due to trapped vortices in niobium cavities, Phys. Rev. ST Accel. Beams 11, 122001 (2008).

[52] J.-M. Vogt, O. Kugeler, and J. Knobloch, High-Q operation of superconducting rf cavities: Potential impact of thermocurrents on the rf surface resistance, Phys. Rev. ST Accel. Beams 18, 042001 (2015).

[53] A. Romanenko and A. Grassellino, Dependence of the microwave surface resistance of superconducting niobium on the magnitude of the rf field, Appl. Phys. Lett. 102, 252603 (2013).

[54] S. Aull, O. Kugeler, and J. Knobloch, Trapped magnetic flux in superconducting niobium samples, Phys. Rev. ST Accel. Beams 15, 062001 (2012).

[55] A. S. Dhavale, P. Dhakal, A. A. Polyanskii, and G. Ciovati, Flux pinning characteristics in cylindrical niobium samples used for superconducting radio frequency cavity fabrication, Supercond. Sci. Technol. 25, 065014 (2012).

[56] Superconductor science and technology special issues: Focus on superconducting rf for accelerators, TBP, http:// iopscience.iop.org/journal/0953-2048/page/Focus-SRF.

[57] T. Matsushita and H. Küpfer, Enhancement of the superconducting critical current from saturation in $\mathrm{Nb}-\mathrm{Ti}$ wire. I, J. Appl. Phys. 63, 5048 (1988).

[58] I. Kaur, Y. Mishin, and W. Gust, Fundamentals of Grain and Interphase Boundary Diffusion, 3rd ed. (John Wiley, Chichester, 1995), p. 512.

[59] G. Eremeev and H. Padamsee, A comparison of large grain and fine grain cavities using thermometry, in Proceedings of the 10th European Particle Accelerator Conference (EPAC 2006), Edinburgh, Scotland, 2006 (JACoW, Geneva, Switzerland, 2006), http://accelconf .web.cern.ch/AccelConf/e06/PAPERS/MOPCH176.PDF. 
[60] P. J. Lee, A. A. Polyanskii, Z.-H. Sung, D. C. Larbalestier, C. Antoine, P. C. Bauer, C. Boffo, and H. T. Edwards Flux penetration into grain boundaries large grain niobium sheet for SRF cavities: Angular sensitivity, AIP Conf. Proc. 927, 113 (2007)

[61] Z.H. Sung et al., An investigation of the influence of grain boundaries on flux penetration in high purity large grain niobium for particle accelerators, in Proceedings of the13th International Workshop on RF Superconductivity (SRF 2007), Beijing, China, 2007 (JACoW, Geneva, Switzerland, 2007), http://accelconf.web.cern.ch/ AccelConf/srf2007/TALKS/WE105_TALK.pdf.

[62] P. J. Lee et al., Grain boundary flux penetration and resistivity in large grain niobium sheet, Physica (Amsterdam) 441C, 126 (2006).

[63] L. Y. Vinnikov, E. Zasavitskii, and S. Moskvin, Critical currents in niobium bicrystals, Sov. Phys. JETP 56, 6 (1982).

[64] G. Zerweck, On pinning of superconducting flux lines by grain boundaries, J. Low Temp. Phys. 42, 1 (1981).

[65] W. Singer et al. Advances in large grain/single crystal SC resonators at DESY, in Proceedings of the 22nd Particle Accelerator Conference, PAC-2007, Albuquerque, NM, USA (IEEE, New York, 2007), http://accelconf.web.cern .ch/AccelConf/p07/PAPERS/THOAKI01.PDF.

[66] P. Kneisel, Progress on large grain and single grain niobium-Ingots and sheet and review of progress on large grain and single grain niobium cavities, in Proceedings of the13th International Workshop on RF Superconductivity (SRF 2007), Beijing, China, 2007 (JACoW, Geneva, Switzerland, 2007), http://accelconf.web.cern .ch/AccelConf/srf2007/PAPERS/TH102.pdf.

[67] P. Kneisel et al. Performance of large grain and single crystal $\mathrm{Nb}$ cavities, in Proceedings of the12th International Workshop on RF Superconductivity (SRF 2005), Ithaca, NY, USA, 2005 (JACoW, Geneva, Switzerland, 2005).

[68] J. Amrit and C. Z. Antoine, Kapitza resistance cooling of single crystal (111) niobium for superconducting rf cavities, Phys. Rev. ST Accel. Beams 13, 023201 (2010).

[69] A. Ermakov, I. Jelezov, X. Singer, W. Singer, G. B. Viswanathan, V. Levit, H. L. Fraser, H. Wen, and M. Spiwek, Physical properties and structure of large grain/ single crystal niobium for superconducting rf cavities, J. Phys. Conf. Ser. 97, 012014 (2008).

[70] G. Ciovati, P. Kneisel, and A. Gurevich, Measurement of the high-field Q drop in a high-purity large-grain niobium cavity for different oxidation processes, Phys. Rev. ST Accel. Beams 10, 062002 (2007).

[71] G. Ciovati et al., Performances of high purity niobium cavities with different grain sizes (Proceedings of the 2006 Linear accelerator conference (LINAC 2006), Knoxville, TE, USA, 2006 (JACoW, Geneva, Switzerland, 2006), http://accelconf.web.cern.ch/AccelConf/106/ PAPERS/TUP033.PDF.

[72] Z-H Sung, P. J. Lee, A. Gurevich, and D. C. Larbalestier, Evidence for preferential flux flow at the grain boundaries of superconducting rf-quality niobium, Supercond. Sci. Technol. 31, 045001 (2018).
[73] A. Gurevich, Multiscale mechanisms of SRF breakdown, Physica (Amsterdam) 441C, 38 (2006).

[74] G. Ciovati and A. Gurevich, Measurement of rf losses due to trapped flux in large-grain niobium cavity, in Proceedings of the13th International Workshop on RF Superconductivity (SRF 2007), Beijing, China, 2007 (JACoW, Geneva, Switzerland, 2007), http://accelconf .web.cern.ch/AccelConf/srf2007/PAPERS/TUP13.pdf.

[75] W. Singer et al., Fabrication of single crystal niobium cavities, in Proceedings of the13th International Workshop on RF Superconductivity (SRF 2007), Beijing, China, 2007 (JACoW, Geneva, Switzerland, 2007), http://accelconf.web.cern.ch/AccelConf/srf2007/HTML/ AUTHOR.HTM.

[76] A. I. Larkin and Y. N. Ovchinnikov, Pinning in type II superconductors, J. Low Temp. Phys. 34, 409 (1979).

[77] H. Kerchner, The statistical summation of weak flux-line pins in type II superconductors, J. Low Temp. Phys. 50, 337 (1983).

[78] T. Matsushita, M. Kiuchi, A. Yamamoto, J. Shimoyama, and K. Kishio, Critical current density and flux pinning in superconducting MgB2, Physica (Amsterdam) 468C, 1833 (2008).

[79] M. Wang et al., Introduction of precisely controlled microstructural defects into SRF cavity niobium sheets and their impact on local superconducting properties, in Proceedings of the 17th Conference on RF superconductivity (SRF 2015), Whistler, BC, Canada, 2015 (JACoW, Geneva, Switzerland, 2015).

[80] Z.-H. Sung, M. Wang, A. A. Polyanskii, C. Santosh, S. Balachandran, C. Compton, D. C. Larbalestier, T. R. Bieler, and P. J. Lee, Development of low angle grain boundaries in lightly deformed superconducting niobium and their influence on hydride distribution and flux perturbation, J. Appl. Phys. 121, 193903 (2017).

[81] S. Chandrasekaran et al., Effect of heat treatment temperature on the thermal conductivity of large grain superconducting niobium parameters, Proceedings of the 15th conference on RF superconductivity (SRF 2011), Chicago, USA, 2011 (JACoW, Geneva, Switzerland, 2011).

[82] A. Narlikar and D. Dew-Hughes, The effect of dislocation configuration on the superconducting properties of niobium and vanadium, Phys. Status Solidi (b) 6, 383 (1964).

[83] C. Antoine, Materials, and Surface Aspects in the Development of SRF Niobium Cavities, EUCARD series on Accelerator Science, edited by R. S. Romaniuk and J. P. Koutchouk (OWPW, Warsaw, Poland, 2012), Vol. 12.

[84] Z.-H. Sung, A. Dzyuba, P. J. Lee, D. C. Larbalestier, and L. D. Cooley, Evidence of incomplete annealing at $800^{\circ} \mathrm{C}$ and the effects of $120^{\circ} \mathrm{C}$ baking on the crystal orientation and the surface superconducting properties of coldworked and chemically polished $\mathrm{Nb}$, Supercond. Sci. Technol. 28, 075003 (2015).

[85] J.A. Good and E. J. Kramer, Deformation-induced anisotropy of the critical current in single crystal niobium, Philos. Mag. 22, 329 (1970).

[86] J. A. Good and E. J. Kramer, Yield and recovery of the flux line lattice in a type II superconductor, Philos. Mag. 24, 339 (1971). 
[87] L. Y. Vinnikov, V. Grigor'ev, and O. Zharikov, Resistive state and pinning in deformed niobium monocrystals, $\mathrm{Zh}$. Eksp. Teor. Fiz. 71, 252 (1976).

[88] C.-P. Chang, The dislocation morphology in deformed and irradiated niobium, Report No. IS-T-763, 1977.

[89] M. Wang et al., Investigation of the effect of strategically selected grain boundaries on superconducting properties of SRF cavity niobium, in Proceedings of the 18th International Conference on $r f$ Superconductivity (SRF'17), Lanzhou, China, 2017 (JACoW, Geneva, Switzerland, 2018), http://accelconf.web.cern.ch/AccelConf/ srf2017/papers/thpb026.pdf.

[90] A. Romanenko et al., Studies of the high field anomalous losses in small and large grain niobium cavities, in Proceedings of the13th International Workshop on RF Superconductivity (SRF 2007), Beijing, China, 2007, (JACoW, Geneva, Switzerland, 2007), http://accelconf .web.cern.ch/AccelConf/srf2007/PAPERS/TUP24.pdf.

[91] C. Herring, The observation of flux line pinning in superconducting foils, Phys. Lett. A 47, 105 (1974).

[92] J. F. Fries, Influence des éléments interstitials (O, C, N) sur le comportement plastique en traction du niobium polycristallin entre $-253 \mathrm{C}$ et $850 \mathrm{C}$, Ph.D. Thesis, Paris XI, Orsay, 1972.

[93] M. Martinello et al., Tailoring surface impurity content to maximize Q-factors of superconducting resonators, in Proceedings of the 7th International Particle Accelerator Conference (IPAC'16), Busan, Korea, 2016 (JACoW, Geneva, Switzerland, 2016), http://accelconf.web.cern .ch/AccelConf/ipac2016/papers/wepmr003.pdf.

[94] M. Martinello, A. Grassellino, M. Checchin, A. Romanenko, O. Melnychuk, D. A. Sergatskov, S. Posen, and J. F. Zasadzinski, Effect of interstitial impurities on the field dependent microwave surface resistance of niobium, Appl. Phys. Lett. 109, 062601 (2016).

[95] A. Romanenko and H. Padamsee, The role of near-surface dislocations in the high magnetic field performance of superconducting niobium cavities, Supercond. Sci. Technol. 23, 045008 (2010).

[96] M. Delheusy, X-ray investigation of Nb/oxide interfaces, Ph.D. Thesis, Orsay/Stuttgart, 2008.

[97] M. Delheusy, A. Stierle, N. Kasper, R. P. Kurta, A. Vlad, H. Dosch, C. Antoine, A. Resta, E. Lundgren, and J. Andersen, X-ray investigation of subsurface interstitial oxygen at Nb/oxide interfaces, Appl. Phys. Lett. 92, 101911 (2008).

[98] L. Torne and G. Thomas, Yielding and plastic flow in niobium, LBNL Report No. UCRL-10515, Lawrence Berkeley National Laboratory, https://escholarship.org/ uc/item/9kx5w22j.

[99] E. Thuneberg, J. Kurkijärvi, and D. Rainer, Pinning of a Vortex Line to a Small Defect in Superconductors, Phys. Rev. Lett. 48, 1853 (1982).

[100] S. Chetri et al., Determination of bulk and surface superconducting properties of N2-doped cold worked, heat treated and electropolished SRF grade niobium, in Proceedings of the 17th International Conference on rf Superconductivity (SRF2015), Whistler, BC, Canada, 2015 (JACoW, Geneva, Switzerland, 2015), http://accelconf .web.cern.ch/AccelConf/SRF2015/papers/mopb052.pdf.
[101] V. Arbet-Engels, C. Benvenuti, S. Calatroni, P. Darriulat, M. A. Peck, A.-M. Valente, and C. A. Van't Hof, Superconducting niobium cavities, a case for the film technology, Nucl. Instrum. Methods Phys. Res., Sect. A 463, 1 (2001).

[102] A. Romanenko, C. J. Edwardson, P. G. Coleman, and P. J. Simpson, The effect of vacancies on the microwave surface resistance of niobium revealed by positron annihilation spectroscopy, Appl. Phys. Lett. 102, 232601 (2013).

[103] B. Visentin, M. F. Barthe, V. Moineau, and P. Desgardin, Involvement of hydrogen-vacancy complexes in the baking effect of niobium cavities, Phys. Rev. ST Accel. Beams 13, 052002 (2010).

[104] A. Romanenko et al., Post-baking losses in electropolished niobium cavities: Cutout studies, in Proceedings of the 5th Workshop on RF Superconductivity (SRF 2011), Chicago, 2011 (JACoW, Geneva, Switzerland, 2011).

[105] L. Embon et al., Probing dynamics and pinning of single vortices in superconductors at nanometer scales, Sci. Rep. 5, 7598 (2015).

[106] B. Bonin and R. W. Röth, Q degradation of niobium cavities due to hydrogen contamination, in Proceedings of the 5th Workshop on RF superconductivity, DESY, Hambourg, Germany, 1991 (JACoW, Geneva, Switzerland, 1991).

[107] C. Z. Antoine et al. The role of atomic hydrogen in Q-degradation of niobium superconducting cavities: The analytical point of view, in Proceedings of the 5th Workshop on RF superconductivity, DESY, Hambourg, Germany 1991 (JACoW, Geneva, Switzerland, 1991).

[108] J. Knobloch, The "Q Disease" in superconducting niobium rf cavities, in Proceedings of the 1st International Workshop on hydrogen in material and vacuum systems, 2002, Newport News, USA, AIP Conf. Proc. Vol. 671 (New York, 2003), pp. 133-150.

[109] C. Z. Antoine, S. Berry, and H. Shou, Hydrogen surface analysis of niobium in function of various electrochemical conditions, in Proceedings of the 11th workshop on RF Superconductivity (SRF 2003), Lübeck, Germany, 2003 (JACoW, Geneva, Switzerland, 2003).

[110] C. Antoine and S. Berry, H in niobium: Origin and method of detection, in Proceedings of the 1st International Workshop on hydrogen in material and vacuum systems, 2002, Newport News, USA, AIP Conf.Proc. Vol. 671 (New York, 2003), pp. 176-189.

[111] B. Roux, A. Chevarier, N. Chevarier, B. Wybourn, C. Antoine, B. Bonin, P. Bosland, and S. Cantacuzene, High resolution hydrogen profiling in superconducting materials by ion beam analysis (ERD-EXB), Vacuum 46, 929 (1995).

[112] B. Aune et al., Degradation of $\mathrm{Nb}$ superconducting rf cavities during cooling time, in Proceedings of the Linear Accelerator Conference (LINAC 1990), Albuquerque, NM, USA, 1990 (JACoW, Geneva, Switzerland, 1990), http:// accelconf.web.cern.ch/AccelConf/190/papers/mo472.pdf.

[113] Errata to reference 109: Read the depths in Table 2 in Angström, not in nm. Figures in the text are correct. 
[114] G. Alefeld and J. Völkl, Hydrogen in metals I, Basic properties, Topics in Applied Physics, edited by H. K. V. Lotsch (Springer-Verlag, Berlin, 1978).

[115] J. A. Rodriguez and R. Kircheim, More evidence for the formation of a dense Cotrell cloud of hydrogen (hydride) at dislocation in niobium and palladium, Scr. Metall. 17, 159 (1983).

[116] H. Wipf, Hydrogen in metals III, Topics in Applied Physics (Springer-Verlag, Berlin, 1997).

[117] J. Č́ížek, I. Procházka, F. Bečvář, R. Kužel, M. Cieslar, G. Brauer, W. Anwand, R. Kirchheim, and A. Pundt, Hydrogen-induced defects in bulk niobium, Phys. Rev. B 69, 224106 (2004).

[118] C. Z. Antoine and R. Crooks, Reducing electropolishing time with chemical-mechanical polishing, Proceedings of the 14th International Conference on RF Superconductivity (SRF 2009), Berlin, Germany 2009, (JACoW, Geneva, Switzerland, 2009), http://accelconf.web.cern .ch/AccelConf/srf2009/html/author.htm.

[119] T. Proslier (private communication).

[120] Y. Trenikhina, A. Romanenko, J. Kwon, J.-M. Zuo, and J. F. Zasadzinski, Nanostructural features degrading the performance of superconducting radio frequency niobium cavities revealed by transmission electron microscopy and electron energy loss spectroscopy, J. Appl. Phys. 117, 154507 (2015).

[121] D. C. Ford, L. D. Cooley, and D. N. Seidman, Firstprinciples calculations of niobium hydride formation in superconducting radio-frequency cavities, Supercond. Sci. Technol. 26, 095002 (2013).

[122] T. Junginger, Review of muSR studies for SRF applications, in 6th International Workshop on Thin films applied to superconducting RF and new ideas (TFSRF 2016) (Jefferson Lab, VA, USA, 2016).

[123] C. Morkel, H. Wipf, and K. Neumaier, Nitrogen-hydrogen interstitial pair in niobium as a new system showing atomic tunneling, Phys. Rev. Lett. 40, 947 (1978).

[124] H. Grabert and H. Wipf, Tunneling of hydrogen in metals, in Festkörperprobleme 30 (Springer, New York, 1990), pp. $1-23$.

[125] D. C. Ford, L. D. Cooley, and D. N. Seidman, Suppression of hydride precipitates in niobium superconducting radio-frequency cavities, Supercond. Sci. Technol. 26, 105003 (2013).

[126] P. Zapp and H. Birnbaum, Mobility of hydrogen around nitrogen and oxygen trapping sites-Phonon assisted tunneling parameters, Acta Metall. 28, 1523 (1980).

[127] G. Cannelli, R. Cantelli, and G. Vertechi, Nonclassical anelastic behavior of hydrogen at liquid helium temperatures in the $\mathrm{Nb}-\mathrm{O}(\mathrm{N})-\mathrm{H}$ and $\mathrm{Nb}-\mathrm{Ti}-\mathrm{H}$ systems, J. LessCommon Met. 88, 335 (1982).

[128] A. Shirley, C. Hall, and N. Prince, Trapping of hydrogen by oxygen and nitrogen impurities in niobium, vanadium and tantalum, Acta Metall. 31, 985 (1983).

[129] P. Hautojarvi, H. Huomo, M. Puska, and A. Vehanen, Vacancy recovery and vacancy-hydrogen interaction in niobium and tantalum studied by positrons, Phys. Rev. B 32, 4326 (1985).

[130] T. Pfiz, R. Messer, and A. Seeger, Nuclear magnetic resonance (NMR) studies of hydrogen tunneling and trapping in niobium, in International Symposium on Metal-Hydrogen Systems-Fundamental and Applications (Stuttgart, Germany, 1988).

[131] B. J. Makenas and H. K. Birnbaum, Phase changes in the niobium-hydrogen system I: Accommodation effects during hydride precipitation, Acta Metall. 28, 979 (1980).

[132] A. Chincarini et al., Statistical approach to XPS analysis: Application to niobium surface treatment, in Proceedings of the 10th Workshop on RF Superconductivity (SRF 2001), Tsukuba, Japan, 2001 (JACoW, Geneva, Switzerland, 2001).

[133] T. Proslier (private communication).

[134] W. M. Albrecht, W. D. Goode, and M. W. Mallett, Reactions in the niobium-hydrogen system, J. Electrochem. Soc. 106, 981 (1959).

[135] S. Isagawa, Influence of $\mathrm{H}$ on $\mathrm{Nb}$ superconducting cavities, J. Appl. Phys. 51, 6010 (1980).

[136] F. Barkov, A. Romanenko, Y. Trenikhina, and A. Grassellino, Precipitation of hydrides in high purity niobium after different treatments, J. Appl. Phys. 114, 164904 (2013).

[137] A. Dzyuba and L. Cooley, Combined effects of cold work and chemical polishing on the absorption and release of hydrogen from SRF cavities inferred from resistance measurements of cavity-grade niobium bars, Supercond. Sci. Technol. 27, 035001 (2014).

[138] W. Espe, Material for high vacuum technology, Metals and Metaloids (Pergamon Press, New York, 1966), Vol. 1.

[139] A. Romanenko, Surface Characterization of Nb Cavity Sections-Understanding the High Field Q-Slope, Ph.D. thesis, Cornell University, Ithaca, 2009.

[140] T. Junginger et al. Low energy muon spin rotation and point contact tunneling applied to niobium films for SRF cavities, in 17th International Conference on RF Superconductivity (SRF2015), Whistler, BC, Canada, 2015 (JACoW, Geneva, Switzerland, 2015).

[141] A. Romanenko, F. Barkov, L. D. Cooley, and A Grassellino, Proximity breakdown of hydrides in superconducting niobium cavities, Supercond. Sci. Technol. 26, 035003 (2013).

[142] A. Romanenko and L. Goncharova, Elastic recoil detection studies of near-surface hydrogen in cavitygrade niobium, Supercond. Sci. Technol. 24, 105017 (2011).

[143] S. Balachandran, Hydrogen distribution and hydride precipitation in SRF Nb revealed by metallographic techniques, Proceedings of the 18th International Conference on RF Superconductivity (SRF 2017), Lanzhou, China, 2017 (JACoW, Geneva, Switzerland, 2017), http://accelconf .web.cern.ch/AccelConf/srf2017/talks/tuxba05_talk.pdf.

[144] P. Dhakal et al., Effect of high temperature heat treatments on the quality factor of a large-grain superconducting radio-frequency niobium cavity, Phys. Rev. ST Accel. Beams 16, 042001 (2013).

[145] Thomas Proslier, M. Kharitonov, M. Pellin, J. Zasadzinski, and Ciovati, Evidence of surface paramagnetism in niobium and consequences for the superconducting cavity surface impedance, IEEE Trans. Appl. Supercond. 21, 2619 (2011).

[146] T. Proslier et al., Tunneling study of SRF cavity-grade niobium, IEEE Trans. Appl. Supercond. 93, 1404 (2008).

[147] C. Z. Antoine (unpublished). 